\title{
Community based medical education: Prospects and challenges
}

\author{
Marahatta SB
}

Assistant Professor, Kathmandu University School of Medical Science, Dhulikhel, Nepal

$\mathrm{M}$ edical education should be responsible towards the community by producing health personnel who respond to the wider health needs of the population they serve. The health care need of the countries varies as per the socioeconomic, demographic, educational status and health care technology and various other factors that affect our livelihood. The medical education of the universities of Nepal has been guided by the ethos of community based medical education with firm foundation on public health orientation. Community based practices have been focused in the curriculum from the beginning year till the end year of medical education. In reality, its time to assess to what extent the community based medical education has instilled the knowledge and attitude in the minds of our graduates to address the health care reality of our country.

There has been a long debate in Nepal and a lot of criticism of Institute of Medicine when the community based medical education was initiated for the first time in Nepal. Should the medical education give more orientation to tertiary /secondary care or primary care? Medical education with secondary/ tertiary care is focused on training medical graduates to assume hospital based role rather than primary health care physicians. In country like Nepal where people are suffering from double burden of communicable diseases and emerging non-communicable diseases attributable to the changes in life-style, medical education needs to be focused towards identifying health problems, appreciating the influence of cultural and socioeconomic factors in determining their health problems, investigating these, and offering rational and realistic solutions.

Community based practices are essential components with the specific aim of developing the student's competencies in understanding community health problems and later enable them to solve these problems working with the community. All field activities should be need-responsive, problem solving, and community oriented, which encourages students to render services while they learn and develop clinical and public health management skills. Community oriented programmes can create more appropriate knowledge, skills, and attitudes; deepen understanding of the whole range of health, illness, and the workings of health and social services; deepen the understanding of social and environmental factors to the causation and prevention of illness; make better use of expertise and availability of staff and patients who are in primary care settings; enhance multidisciplinary working; offer a broad range of learning opportunities; and increase recruitment to primary care as well.

One of the most problematic aspects of the current community based medical education is the gap between knowing and practicing community health care, between competence and performance in community settings; and an ability to apply knowledge using appropriate skills, with a professional, caring attitude towards primary care. Realists might argue that the performance competence gap is a manifestation of lacking of knowhow to practice community care. Medical students need to be given opportunity towards individual education and development, time to explore the important field of informatics, time to review health care delivery of the country and work in multidisciplinary teams. They also need time to give adequate, or even generous, support to the co-workers in health centres, and time to reflect on what they have achieved. A further prescription might be to ensure that they are able to act as a positive role model in community to enjoy work and to feel themselves that they can make a contribution to their profession and the health of the nation. For national healthcare systems to improve, the training of health professionals must be reoriented, to enable them to serve satisfactorily in areas with dense populations and high prevalence of disease. The focus of the community based medical education need to be designed to enable our graduates to serve in rural part of the country and address their health care needs. The present epidemic of diarrhoea / Cholera in the mid-western part of Nepal and lacking of the sound measures to tackle with the problem reflects that the public health orientation is often lacking in our medical professionals. Capacity building for public health research in developing countries is therefore needed, and this need is greatest in county like ours. This can be solved through experts and consultants, produced through residencies in community health. They can undertake research in communities, thereby generating 
research capacity in addressing the disease rampant in these areas. Community based medical education and integrated curriculum in undergraduate and professional training will add to the process.

Medical schools need to continually address on target areas with continual follow up of the work and focus on addressing health care needs along with the academic pursuit. Innovative health interventions need to be designed and applied as per the particular circumstances. To strengthen community based medical education medical institutions need to be accountable and must design innovative intervention strategy to address the health problems using the available resources with active participation of the community. 